\title{
Artrodesis cervical anterior mediante implante de tantalio. Resultados clínicos y radiológicos
}

\author{
C. Vicario; F. Lopez-Oliva; T. Sánchez-Lorente; M. Zimmermann*; J.J. Asenjo-Siguero; F. Ladero y A. Ibarzábal
}

Unidad de columna, Centro de Rehabilitación FREMAP, Majadahonda, Madrid. *Servicio de Salud Laboral. Instituto de Salud Publica. Comunidad de Madrid.

\section{Resumen}

La discectomía cervical anterior con artrodesis intersomática (DCAA) es una técnica quirúrgica ampliamente aceptada en el tratamiento de la enfermedad discal cervical. Recientemente se han introducido con esta indicación las cajas de tantalio debido a sus excelentes propiedades mecánicas y biológicas.

Presentamos los resultados iniciales clínicos y radiológicos de un estudio prospectivo de 24 pacientes intervenidos en nuestro servicio tras el empleo de cajas de tantalio en la DCAA. La evaluación clínica pre y postoperatoria se realizó mediante cuestionario que incluía una Escala Visual Analógica de dolor, el Î́ndice de Oswestry, y la Escala de Zung. Los resultados fueron clasificados mediante los criterios de Odom. La evaluación radiológica incluyó radiografías dinámicas en las que se midieron las variaciones del ángulo de Cobb y la distancia interespinosa. Postoperatoriamente los pacientes fueron revisados en el tercer mes y al año de la intervención.

Todos los parámetros clínicos mejoraron de forma estadísticamente significativa. Según con los criterios de Odom, el $\mathbf{7 5 \%}$ de los casos fueron considerados como excelentes o buenos. Sólo se confirmó clínica y radiológicamente un caso de pseudoartrosis. No se observaron diferencias significativas en las revisiones al año y a los 3 meses.

Conclusión: Las cajas de tantalio constituyen un implante prometedor $\mathrm{y}$ pensamos que útil entre las diversas opciones quirúrgicas para la DCAA, por sus buenos resultados clínicos, por la no necesidad de autoinjerto, y por su excelente compatibilidad con la resonancia nuclear magnética.

PALABRAS CLAVE: Discectomía anterior cervical. Tantalio. Hedrocel. Artrosis cervical. Hernia discal cervical.

Recibido:19-11-04. Aceptado: 18-05-05 $\overline{\text { Anterior cervical fusion with tantalum interbody }}$ implants. Clinical and radiological results in a prospective study

Summary

Anterior cervical discectomy and interbody fusion (ACDF) is a widely accepted surgical technique in the treatment of cervical disc disease. Tantalum cages have been recently introduced in spine surgery for interbody fusion because of the advantages of their mechanical properties.

We present the results of a prospective clinical and radiological study on 24 consecutive patients who underwent an ACDF with tantalum cages. Clinical evaluation was assessed preoperatively and after surgery by a questionnaire that included a Visual Analogic Scale (VAS) of neck and arm pain, the Oswestry Dissability Index and the Zung Depression Scale. Results were classified by Odom's criteria. Radiological evaluation included flexion-extension X-rays, and changes in distance between spinous processes and Cobb angle were measured. Postoperatively patients were reviewed 3 and 12 months after surgery.

A statisitical significative improvement in all clinical data was reported. According to Odom's criteria in $75 \%$ of patients the results were considered like excellent or good. Only one case of radiological and clinical pseudoarthrosis was confirmed. No significative differences were reported 3 and 12 months after surgery.

Tantalum cages are a very promising and usefull alternative among implants available for ACDF. Compatibility with MRI postoperative studies and the unnecessariness of autograft are some of their advantages.

KEY WORDS: Anterior cervical discectomy. Tantalum. Hedrocel. Cervical spondylosis. Cervical disc herniation.

Abreviaturas. ACDF: anterior cervical discectomy and interbody fusion. DCAA: discectomía cervical anterior con artrodesis intersomática. EVA: escala visual analógica del dolor. VAS: visual analogic scale. 


\section{Introducción}

La discectomía cervical anterior con artrodesis intersomática (DCAA) es una técnica quirúrgica ampliamente aceptada para el tratamiento de la enfermedad discal cervical ${ }^{3}$. Desde 1958, cuando fue inicialmente descrita por Smith y Robinson ${ }^{18}$ y Cloward ${ }^{7}$, se han descrito numerosas variantes técnicas ${ }^{17}$, y se han empleado diversos tipos de implantes e injertos ${ }^{13}$. Con la mayoría de ellas se han obtenido buenos resultados en cuanto a mejoría del dolor y la función ${ }^{9,12,16,23}$.

Entre los diversos implantes disponibles para la artrodesis anterior cervical, las cajas de tantalio han sido recientemente introducidas. El metal trabecular es un biomaterial compuesto habitualmente de tantalio (Ta), su porosidad es del $80-85 \%$ y el tamaño medio de su poro es de aproximadamente 550 micras. Es un material muy receptivo para el crecimiento tanto de tejidos blandos como óseos y su módulo de elasticidad está entre los del hueso cortical y esponjoso ${ }^{5,10}$.

Se han publicado muy pocos datos acerca de sus resultados clínicos y/o radiológicos sobre el empleo de cajas de tantalio en la DCAA. Hasta donde tenemos conocimiento, únicamente Wigfield et $\mathrm{a}^{22}$ han publicado su experiencia, y detuvieron su estudio por la presencia de imágenes, en el postoperatorio inmediato, de radiolucencia en las placas limitantes de la vértebra inferior en los pacientes a los que se les implantaron cajas porosas de tantalio para la artrodesis cervical, aunque a los 12 meses estas líneas habían desaparecido y en todos los casos se consideraron fusionados. El principal objetivo de este estudio es describir los resultados clínicos y radiológicos de nuestra experiencia en la DCAA con implantes de tantalio.

\section{Material y métodos}

Se ha realizado un estudio prospectivo de 24 casos consecutivos en los que se realizó una DCAA en uno o dos niveles con cajas de tantalio en nuestro servicio.

17 pacientes $(70,8 \%)$ eran hombres y $7(29,1 \%)$ mujeres. Su edad media fue de 41,8 (DS: 8). En todos los casos el tratamiento quirúrgico se indicó tras el fracaso de un tratamiento conservador realizado durante una media de 5,3 meses (DS: 2.8). Antes de la cirugía, todos los pacientes referían braquialgia y el $75 \%$ cervicalgia significativa. Los hallazgos en la resonancia nuclear magnética (RMN) reflejaban un cuadro de hernia discal en el 62,5\%; mientras que en el resto $(37,5 \%)$, se trataba fundamentalmente de cambios degenerativos.

14 casos estaban considerados, administrativamente, como accidentes laborales, con las implicaciones que ello tiene en el sistema de compensaciones económicas. Los otros 10 pacientes fueron considerados como contingencias

\begin{tabular}{c|c}
\hline \multicolumn{2}{c}{ Tabla 1 } \\
Niveles fusionados \\
\hline Niveles fusionados & Número \\
\hline C5-C7 & 9 \\
\hline C5-C6 & 7 \\
\hline C6-C7 & 3 \\
\hline C4-C5 & 2 \\
\hline C4-C6 & 2 \\
\hline C7-D1 & 1 \\
\hline
\end{tabular}

comunes. En cuanto a la intensidad del esfuerzo del trabajo previo a la cirugía, en 4 casos $(16,7 \%)$ fue considerado como muy demandante.

Preoperatoriamente, se pidió consentimiento informado a los pacientes para participar en el estudio y no hubo ninguna negativa. La evaluación preoperatoria consistió en un cuestionario que incluía una Escala Visual Analógica de dolor (EVA), el Índice de Oswestry ${ }^{8}$, y la Escala de Depresión de Zung ${ }^{24}$. El Índice de Oswestry es un cuestionario inicialmente diseñado para medir el grado de discapacidad en pacientes con lumbalgia, pero se ha usado con éxito en pacientes tras una $\mathrm{DCAA}^{23}$.

La cirugía se realizó en todos los casos a través de un abordaje anterior. En todos los casos se realizó una discectomía completa y resección de los osteofitos posteriores a demanda. Posteriormente se procedió a una decorticación suave de las placas limitantes de ambas vértebras y tras comprobar radiológicamente las dimensiones del implante de prueba se colocó el definitivo (Hedrocel ${ }^{\circledR}$, Implex, Allendale, NJ). En ningún caso se realizó el labrado de una mortaja para alojar el implante. Tampoco se consideró necesario que alcanzara el ligamento común posterior, porque la artrodesis con este tipo de implante se produce por crecimiento óseo a través del mismo $^{2}$. En 13 pacientes $(54,8 \%)$ se realizó una DCAA en un nivel con cajas de tantalio; en el resto se artrodesaron 2 niveles. En la Tabla 1 se resumen los niveles fusionados.

Tras el postoperatorio inmediato, los pacientes fueron revisados en consultas externas y se realizaron radiografías mensuales. La evaluación clínica y radiológica inicial se realizó a los 3 meses postoperatoriamente. Posteriormente se repitió al año. En ambas revisiones, para la evaluación clínica, los pacientes rellenaron un nuevo formulario que incluía una EVA de dolor cervical y en el brazo, el Índice de Oswestry y la Escala de Zung. Los resultados clínicos también fueron evaluados con los criterios de Odom, que son un sistema de gradación de los mismos en excelentes, buenos, regulares y malos ${ }^{15}$. Para la evaluación radiológica, se realizaron radiografías anteroposterior, lateral y dinámicas. En los controles radiológicos iniciales se prestó especial aten- 

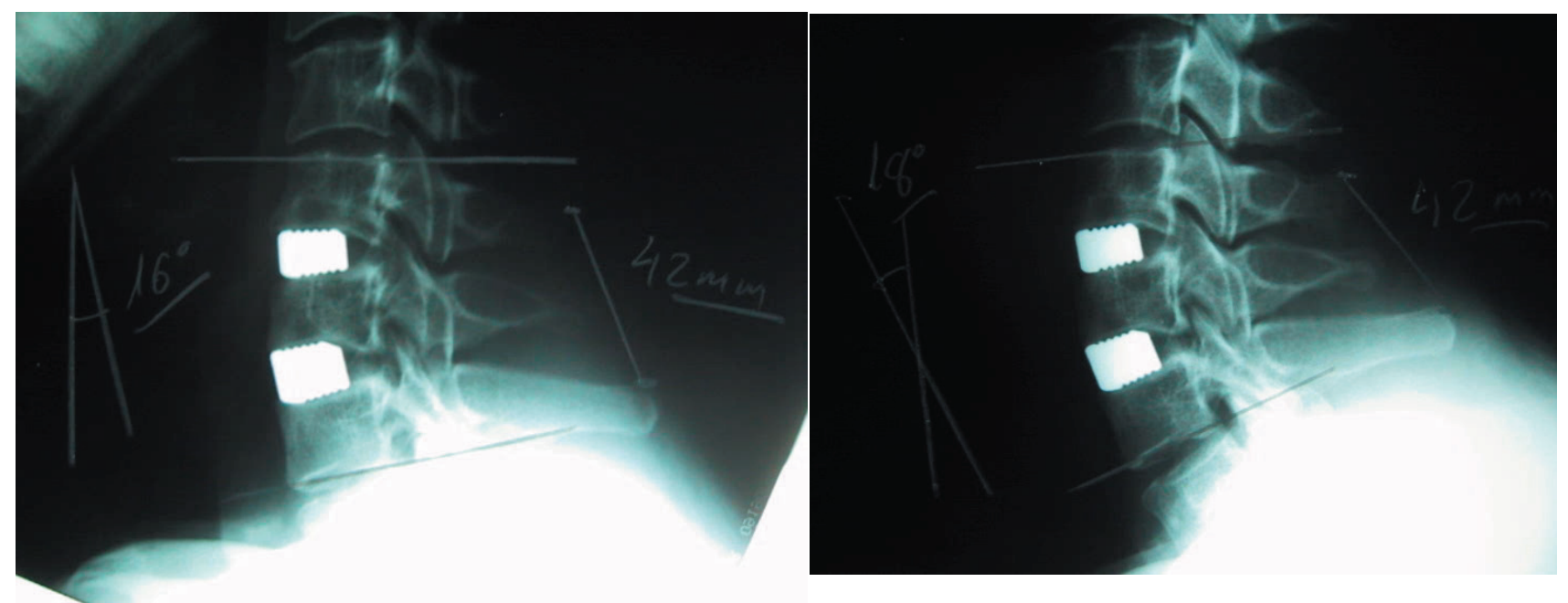

Figura 1. Radiografias dinámicas y medidas de la diferencia entre las distancias interespinosas y el ángulo de Cobb

ción a la posible aparición de imágenes de radiolucencia en las placas limitantes inferiores, que podrían habernos obligado a detener el estudio ${ }^{22}$. Se consideraron como criterios de consolidación radiológica los propuestos por Cannada et $\mathrm{al}^{4}$ : Presencia de trabéculas óseas maduras y remodeladas, puenteando el espacio discal. En las radiografías dinámicas se midieron los cambios en la distancia interespinosa y en el ángulo de Cobb, en los niveles artrodesados (Figura 1). En los pacientes intervenidos a 2 niveles, las medidas fueron realizadas para ambos niveles y los valores absolutos se dividieron por 2 para obtener su valor medio.

El análisis estadístico se realizó mediante los test ChiCuadrado y de Fisher para variantes cualitativas, mediante tests de comparación de medias para variantes dependientes y por el test de comparación de medias de KurskalWallis. El nivel de significación estadística se estableció para $\mathrm{p}<0,05$.

\section{Resultados}

\section{Periodo preoperatorio}

La media en la EVA preoperatoria fue de 6,92 (Rango 4-10) y cuando fue comparada con otras variables (sexo, número de niveles fusionados, etc), no se encontró ninguna relación estadísticamente significativa.

La media del Índice de Oswestry fue de 49,46 (Rango 24-78). Fue estadísticamente mayor $(\mathrm{p}=0.03)$ en los pacientes que fueron sometidos a una DCAA de un nivel (media 54,77) que en los de 2 niveles (media 43,18). No se encontraron otras relaciones estadísticamente significativas, aunque sí cabe destacar un valor mayor $(\mathrm{p}=0,068)$ en los casos de hernia discal (media 53,27) que en aquéllos en los que predominaban los cambios degenerativos (media
43,11).

La media de la Escala de Depresión de Zung fue de 23,33. Fue la variante menos homogénea de las estudiadas, ya que su rango varió entre 3 y 45 puntos. Sin embargo, ninguno de los pacientes podía ser considerado como depresivo, ya que esta circunstancia sucede si el valor de la escala es superior a 55 puntos $^{24}$. Su valor total fue significativamente mayor $(\mathrm{p}=0.007)$ en pacientes que tenían un trabajo de alta o media demanda física (media $28,26)$ que aquéllos cuyo trabajo era de baja demanda física (media 15,6). También fue superior $(\mathrm{p}=0.43$ ) en las mujeres que en los hombres, y en los pacientes que recibieron cirugía en un solo nivel $(p=0.034)$. $Y$, aunque sin significación estadística, también fue mayor en los pacientes que tenían hernia discal que en los que presentaban cambios degenerativos $(\mathrm{p}=0.63)$.

\section{Periodo perioperatorio}

No se han recogido complicaciones intraoperatorias significativas. Se empleó un collarín blando en los pacientes que recibieron una cirugía a 2 niveles durante una media de 4,9 semanas.

Postquirúrgicamente, un paciente presentó un cuadro de disfagia intensa y persistente, a pesar de que no se encontraron alteraciones en el tránsito esofágico realizado. Sus síntomas mejoraron tras unos días de tratamiento conservador. Un paciente al que se le realizó una DCAA a un solo nivel presentó en el postoperatorio inmediato un cuadro de dolor radicular C7 intenso; se le realizó una RMN y no se encontró ninguna alteración significativa y el cuadro de dolor mejoró tras 3 semanas de tratamiento farmacológico. No se han recogido otras complicaciones postoperatorias significativas. 
Tabla 2

Comparación de los valores de la EVA, del Índice de Oswestry y de la Escala de Zung preoperatorios, a los 3 y a los 12 meses. Se puede observar una mejoría estadísticamente significativa en los 3 parámetros

\begin{tabular}{lllclr}
\hline & Media & DS & Diferencia media & t & p \\
\hline EVA Preop & 6,92 & 1,61 & & & \\
\hline EVA 3 Meses & 2,63 & 1,93 & 4,29 & 8,49 & $\mathbf{0 , 0 0 0}$ \\
\hline EVA 12 Meses & 2,38 & 1,84 & 4,54 & 9,52 & $\mathbf{0 , 0 0 0}$ \\
\hline & & & & & \\
\hline Oswestry Preop & 49,46 & 13,27 & & & \\
\hline Oswestry 3 Meses & 21,00 & 16,68 & 28,45 & 10,48 & $\mathbf{0 , 0 0 0}$ \\
\hline Oswestry 12 Meses & 21,82 & 17,02 & 27,64 & 9,26 & $\mathbf{0 , 0 0 0}$ \\
\hline Zung Preop & & & & & \\
\hline Zung 3 Meses & 23,33 & 12,53 & & & \\
\hline Zung 12 Meses & 16,58 & 11,00 & 6,75 & 9,73 & $\mathbf{0 , 0 0 0}$ \\
\hline
\end{tabular}

\section{Evaluación clínica postoperatoria}

Los pacientes fueron evaluados a los 3 y a los 12 meses tras la cirugía, según los criterios de $\operatorname{Odom}^{15}$. La revisión a 12 meses no se pudo realizar en 2 pacientes que no acudieron a las consultas. A los 3 meses, 6 casos (25\%) fueron excelentes, $12(50 \%)$ buenos, $5(20.8 \%)$ regulares, y 1 malo (4.2\%). De los casos perdidos uno fue considerado como excelente y otro como regular. Estas proporciones se mantuvieron a los 12 meses, cuando hubo 7 casos excelentes $(31,8 \%), 9$ buenos $(40,9 \%), 5$ regulares $(22,7 \%)$ y 1 malo $(4,5 \%)$. Cabe destacar que 3 de los casos regulares a los 12 meses se trataban de accidentes de trabajo y estaban inmersos en algún tipo de reclamación de carácter laboral. No se encontraron relaciones estadísticamente significativas cuando se compararon estos datos con otras variable preoperatorias, aunque si cabe decir que los resultados buenos o excelentes fueron más frecuentes entre los pacientes más jóvenes, en aquellos clasificados como contingencias comunes y en los diagnosticados de enfermedad degenerativa.

El valor medio de la VAS a los 3 meses fue de 2,63 (rango 0-6), y a los 12 de 2,38 (rango 0-6). La media del Índice de Discapacidad de Oswestry a los 3 meses fue de 21 puntos (rango 3-52), y a los 12 de 21,8 (rango 5-49). La media de la Escala de Depresión de Zung a los 3 meses fue de 16,58 (rango 3-37) y a los 12 de 14,26 (rango 3-28). Cuando estos datos se compararon con los preoperatorios se observó una mejoría estadísticamente muy significativa tanto a los 3 como a los 12 meses $(p=0.00)$ (Tabla $2)$. Cuando esta mejoría clínica se relacionó con otras variantes preoperatorias no se encontraron diferencias estadísticamente significativas, de modo que no se han podido establecer factores pronósticos positivos o negativos. Únicamente cabe destacar que los varones tuvieron una casi significativa mejoría a los 12 meses en los valores de la EVA (diferencia media de 4,9 en hombres frente a 2,8 en mujeres; $\mathrm{p}=0.06)$.

Cuatro pacientes $(16.7 \%)$ no retornaron a sus trabajos habituales (incapacidad permanente total), 3 de los cuales fueron considerados inicialmente como de alta demanda física. Entre los casos considerados como accidente de trabajo, otros 4 pacientes fueron considerados como una incapacidad permanente parcial. Tres de estos pacientes al final del estudio estaban inmersos en algún tipo de demanda de carácter laboral; en ninguno de ellos la RMN demostró la presencia de alteraciones significativas (Figura 2). Finalmente, otros 3 pacientes recibieron una compensación económica en forma de baremo.

\section{Evaluación radiológica postoperatoria}

No se encontró en ningún caso una radiolucencia de las placas limitantes en los espacios intervenidos en los controles radiológicos mensuales realizados. En la revisión radiológica a los 3 meses la fusión definida como la presencia de trabéculas óseas maduras y remodeladas puenteando el espacio discal ${ }^{4}$ (Figura 3) se observó en todos los casos salvo en 3 en una media de 12,9 semanas. En uno de estos 3 casos el cambio en la distancia entre espinosas fue de $8 \mathrm{~mm}$ y el cambio en el ángulo de Cobb de $10^{\circ}$; es el único paciente considerado como mal resultados según los criterios de Odom y diagnosticado con seguridad de pseudoartrosis, por lo que fue programado para una reintervención. Los otros 2 pacientes en los que no se observaron signos evidentes radiológicos de consolidación a los 3 meses tenían 

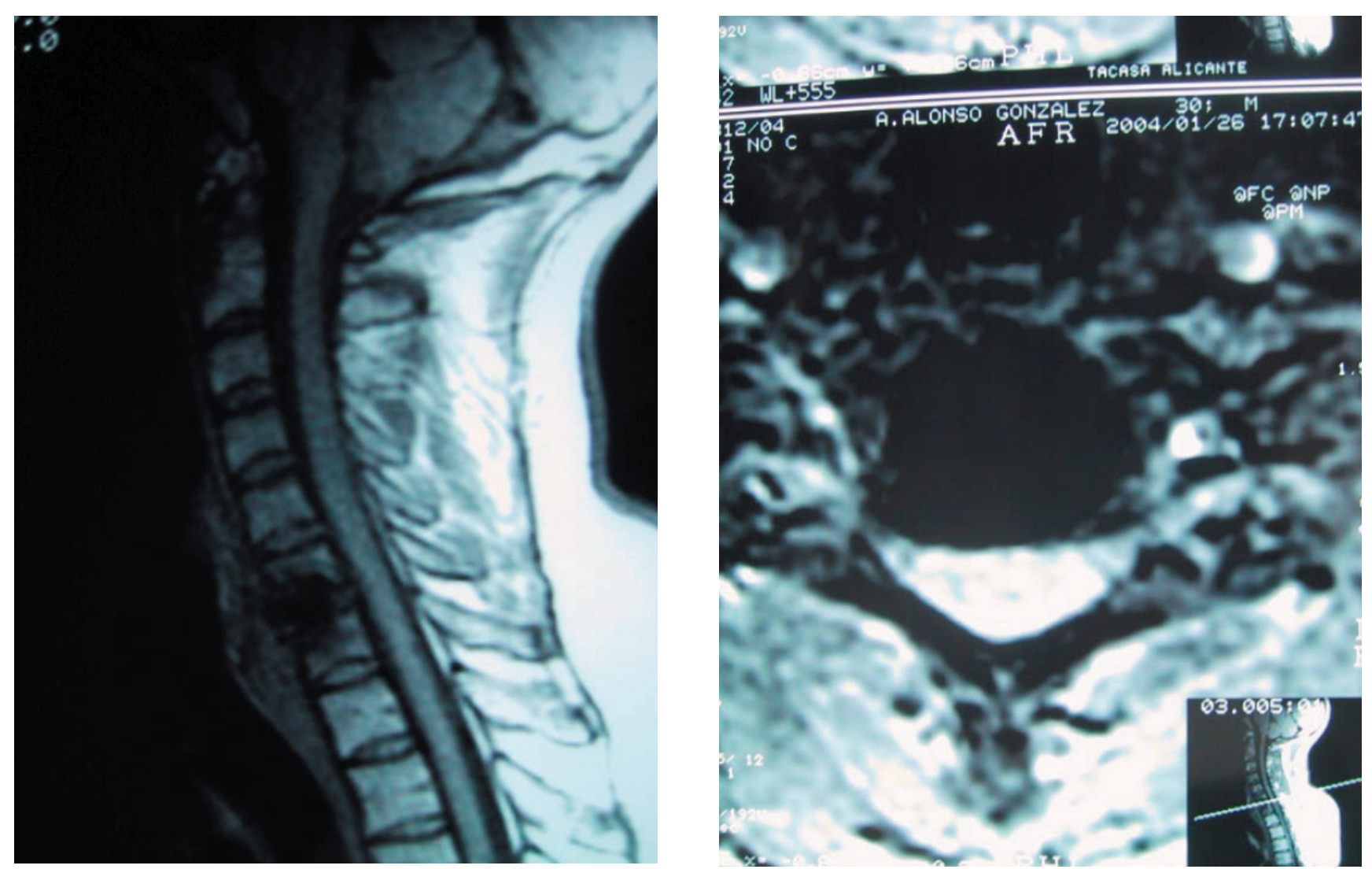

Figura 2. RMN 3 meses tras la cirugía en un paciente con dolor cervical e inmerso en procedimiento legal por demanda con respecto al grado de incapacidad laboral. No se observan alteraciones significativas.

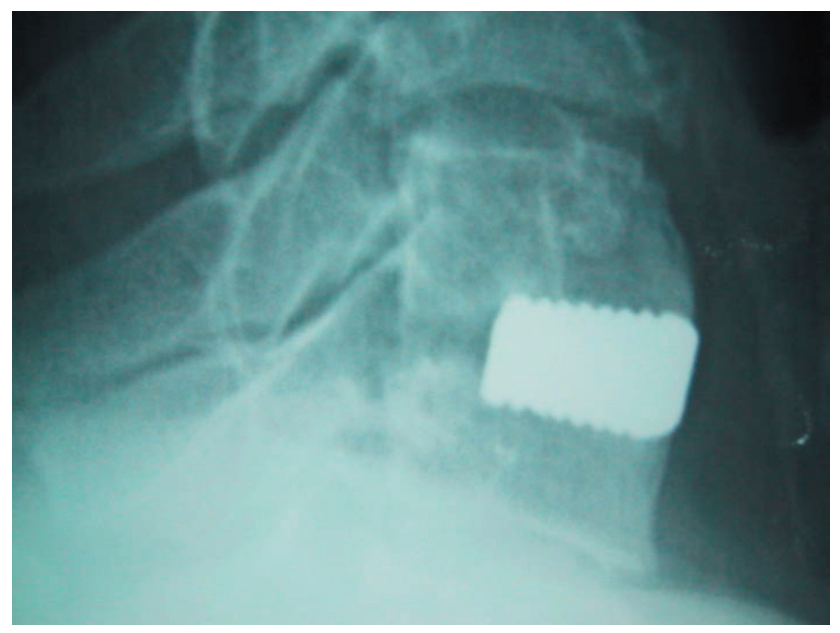

Figura 3. Presencia de trabéculas óseas maduras y remodeladas puenteando el espacio discal 12 semanas tras la intervención.

menos de $2 \mathrm{~mm}$ o $2^{\circ}$ de diferencia en la distancia entre las espinosas o en el ángulo de Cobb respectivamente y por tanto no fueron diagnosticados de pseudoartrosis. Estos 2 casos, en la revisión a los 12 meses, ya presentaban signos radiológicos de consolidación (Figura 4).
Además del caso seguro de pseudoartrosis, se pudo observar a los 3 meses un cambio en la distancia entre las espinosas mayor de $2 \mathrm{~mm}$ en otro paciente, que clínicamente fue clasificado como buen resultado según los criterios de Odom, y retornó a su actividad laboral previa, y por tanto no fue considerado como un caso de pseudoartrosis. En cuanto a la medida de las variaciones del ángulo de Cobb, en otros 3 pacientes ésta fue mayor de $2^{\circ}$, y todos los casos fueron pacientes sometidos a cirugía a 2 niveles. Dos de ellos fueron considerados clínicamente como buenos resultados, y a los 12 meses la variación del ángulo era menor de $2^{\circ}$; mientras que el tercero fue considerado como regular, tenía menos de $1 \mathrm{~mm}$ en los cambios de la distancia interespinosa y rechazó la posibilidad de reintervención. Precisamente este fue uno de los pacientes que no acudió a la revisión a los 12 meses.

\section{Discusión}

La DCAA ha sido un tratamiento quirúrgico satisfactorio de las hernias discales cervicales y de las enfermedades degenerativas de la columna cervical desde los años $50^{1,7,17,18}$. El empleo de injerto óseo autólogo ha sido considerado como el método estándar para alcanzar 


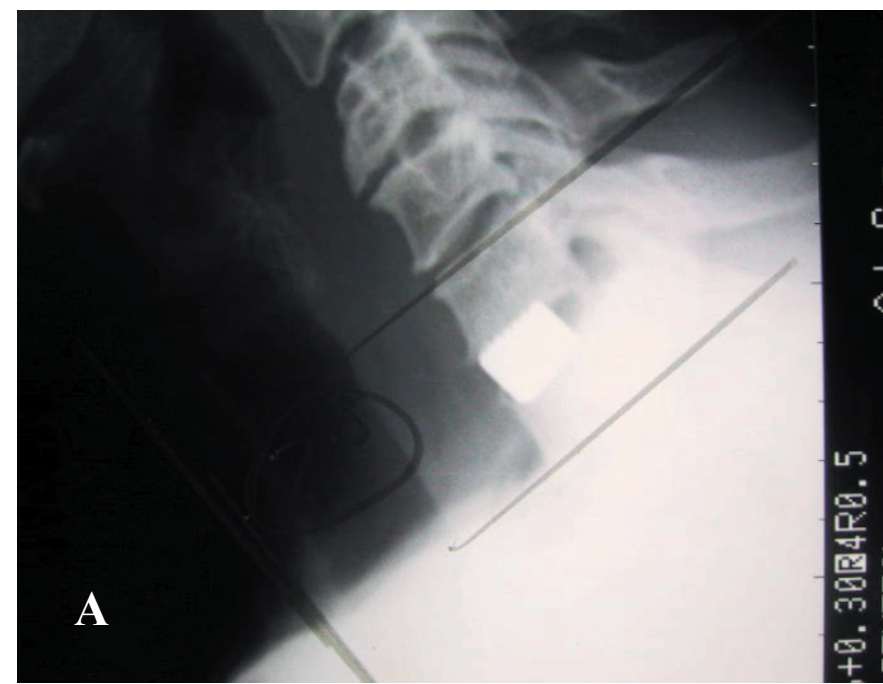

Figura 4. A). Uno de los casos que a los 3 meses no se consideró como consolidado, aunque en las radiografías dinámicas no se observaron criterios de pseudoartrosis.

B). Radiografía a los 12 meses en la que se aprecian criterios de consolidación.

una fusión estable; sin embargo en la literatura se han recogido complicaciones como la morbilidad del sitio donante, o el colapso, extrusión o fracaso de la fijación del injerto ${ }^{6,12,13}$. Ello ha provocado que se desarrollen diferentes técnicas quirúrgicas e implantes para resolver estos problemas $^{9,12,13,20,23}$. En nuestro centro, el empleo de autoinjerto o hueso liofilizado fue abandonado a partir de 1988 cuando encontramos mejores resultados y menor morbilidad con la implantación del dispositivo BAK-C en la DCAA ${ }^{12}$.

En el mercado han aparecido numerosos implantes para su utilización en la DCAA, entre ellos creemos destaca el tantalio ya que sus características físicas resultan muy adecuadas para la fusión cervical anterior ${ }^{5,10}$. A pesar de ello, no existen en la literatura apenas datos sobre su empleo en este tipo de técnicas. Sólo Wigfield et $\mathrm{al}^{22}$ han comunicado su experiencia, pero refieren que tuvieron que detener su estudio prospectivo por la aparición de líneas de radiolucencia inferiores en pacientes tratados con implantes porosos de tantalio. A pesar de todo, en este estudio se obtuvieron resultados clínicos y radiológicos similares a los obtenidos en los pacientes tratados con autoinjerto esponjoso al cabo de 2 años. Ese ha sido uno de los motivos fundamentales que nos impulsó a realizar el presente estudio.

En resumen, nuestra experiencia con el empleo de cajas de tantalio en la DCAA ha proporcionado unos buenos resultados clínicos y radiológicos con muy escasas complicaciones. Por un lado, no hemos encontrado ningún caso de radiolucencia de las placas limitantes inferiores como describieron Wigfield et $\mathrm{al}^{22}$; por otro lado, el alivio sintomático puede ser considerado como muy satisfactorio,

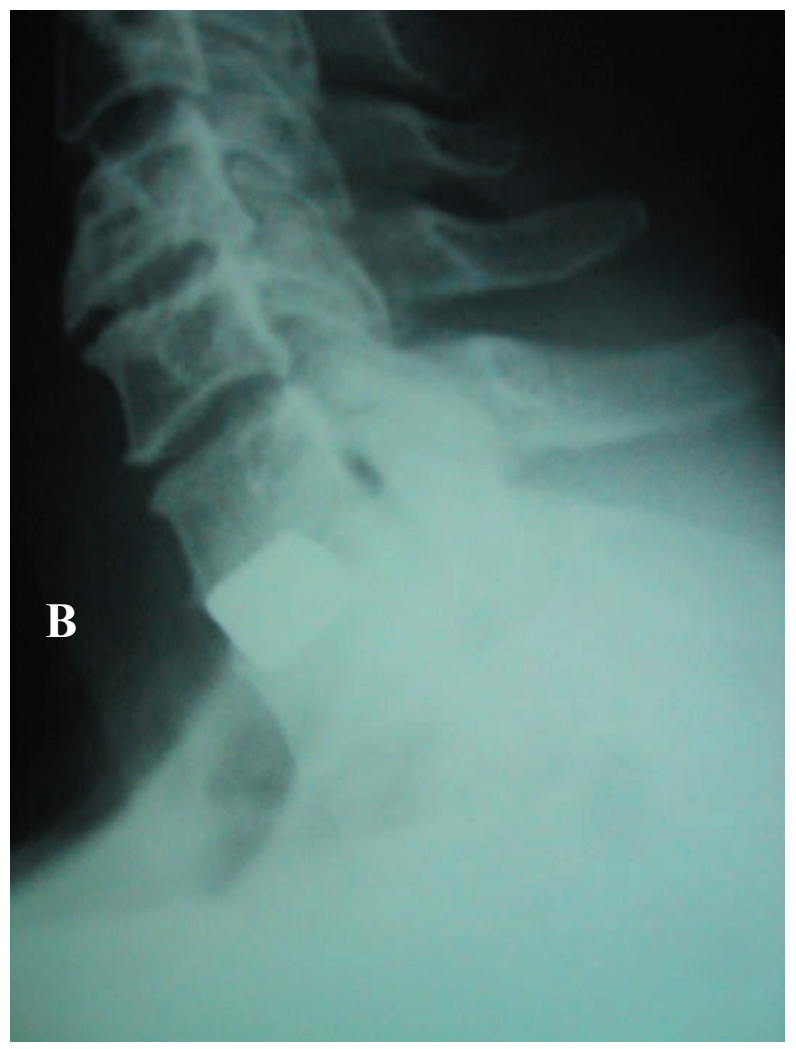

teniendo en cuenta especialmente que un porcentaje importante de pacientes (58\%) eran considerados como accidentes de trabajo, con lo que ello implica en el aspecto de las compensaciones económicas y en el empeoramiento de los resultados finales ${ }^{14}$, especialmente en los 3 casos pendientes de un proceso de reclamación económica (los 3 fueron considerados como regulares). Por último, debemos destacar que no se han encontrado complicaciones radiológicas significativas.

Aparte de estas cuestiones, creemos que se deben hacer diversas consideraciones. En primer lugar, el diagnóstico de pseudoartrosis no puede ser realizado solamente teniendo en cuenta los hallazgos radiológicos ya que algunos pacientes pueden presentar datos radiológicos de falta de unión a la vez que refieren un buen resultado clínico y funcional, $\mathrm{y}$ en quienes, por tanto, no se debe plantear una solución quirúrgica al problema de consolidación radiológica. Sin embargo, en estos pacientes se debe ser especialmente escrupuloso en el seguimiento clínico y radiológico a medio y largo plazo para detectar posibles complicaciones precozmente.

En segundo lugar, creemos que una de las grandes ventajas que tiene el tantalio frente a otros tipos de implantes es su excelente compatibilidad con los estudios de RMN postoperatorios, proporcionando imágenes con muy escasos artefactos ${ }^{11,23}$ (Figura 2). Una de las principales implicaciones que tiene esta excelente compatibilidad es 
el adecuado estudio postquirúrgico de pacientes con síntomas de dolor e incapacidad, en ausencia de alteraciones radiológicas y con problemas médico-legales en cuanto a compensaciones económicas en los casos de accidentes laborales.

En tercer lugar, el empleo de implantes de tantalio evita que sea necesario añadir cualquier tipo de autoinjerto o sustituto óseo para favorecer la artrodesis, ello hace que la técnica sea más sencilla y menos expuesta a variaciones individuales como cuando se emplean otro tipo de implantes.

En cuarto y último lugar, el hecho de que el empleo de collarín no sea imprescindible en el manejo postoperatorio, al menos en los pacientes sometidos a cirugía en un nivel, es una ventaja importante con respecto a otras técnicas. Además podemos añadir que debido a esta buena experiencia, en la actualidad tampoco lo estamos empleando en pacientes sometidos a una DCAA a 2 niveles, aunque estos casos no han sido incluidos en el presente estudio.

Para terminar, creemos que aunque los resultados de esta técnica han sido muy satisfactorios y prometedores, aún se debe completar el estudio de este grupo de pacientes a largo plazo, y determinar cuál es su evolución al menos durante los primeros 2 años, especialmente en aquellos casos que a pesar de haber tenido un resultado clínico satisfactorio, en los controles radiológicos existe algún dato de inestabilidad.

\section{Conclusiones}

Los resultados del empleo de cajas de tantalio en la DCAA han sido muy satisfactorios tanto clínica como radiológicamente y aunque está por determinar la evolución a largo plazo de estos pacientes, creemos que este tipo de implantes aporta algunas ventajas sobre otros, debido por ejemplo a su excelente compatibilidad con estudios postoperatorios de RMN y a que no resulta necesaria la adición de autoinjerto o sustitutos óseos para favorecer la consolidación.

Para la realización de este trabajo no ha existido ninguna relación económica con la firma comercial que fabrica $y$ vende los implantes a los que hace referencia este artículo.

\section{Bibliografía}

1. Bailey, R.W., Badgley, C.E.: Stabilization of the cervical spine by anterior fusion. J Bone Joint Surg- A. 1960; 42-A: 565-594.

2. Bobyn, J.D., Satcakpool, GJ., Hacking, S.A., Tanzer, M, Krygier, J.J.: Characteristics of bone ingrowth and interface of a new porous tantalum biomaterial. J Bone Joint Surg- B. 1999; 81-B: 907-914.
3. Bohlman, H.H.: Cervical spondylosis and mielopathy. Instr Course Lect 1995; 44: 81-97.

4. Cannada, L.K., Scherping, S.C., Yoo, J.U., Jones, P.K., and Emery, S.E.: Pseudoarthrosis of the cervical spine: a comparison of radiographic diagnostic measures. Spine 2003; 28 : 46-51.

5. Christie, M.J.: Clinical applications of Trabecular Metal. Am J Orthop 2002; 31: 219-220.

6. Clements, D.H., O'Leary, P.F.: Anterior cervical discectomy and fusion. Spine 1990; 15: 1023-1025.

7. Cloward, R.B.: The anterior approach for removal of ruptured cervical disks. J Neurosurg 1958; 15: 602-616.

8. Fairbank, J.C.T., Couper, J., Davies, J.B., O’Brien, J.P.: The Oswestry low back pain disability questionaire. Physiotherapy 1980; 66: 271-273.

9. Huckell, C.B.: Clinical outcomes after cervical spine fusion. Orthop Clin North Am 1998; 29: 787-799.

10. Jacofsky, D., Hanssen, A., Ladero Morales, F.: Metal trabecular. Posibilidades futuras en cirugía reconstructiva: Patología Aparato Locomotor 2003; 1: 159-163.

11. Levi, A.D., Choi, W.G., Keller, P.J., et al.: The radiographic and imaging characteristics of porous tantalum implants within the human cervical spine. Spine 1998; 23: 1245-1250.

12. López-Oliva Muñoz, F., Garcia de la Heras, B., Concejero Lopez, V., Asenjo Siguero, J.J.: Comparison of three techniques of anterior fusion in single-level cervical disc herniation. Eur Spine J 1998; 7: 512-516.

13. M. de la Torre, Martínez Quiñones, J.V. Escobar, R. Rodríguez, D.: Artrodesis cervical anterior con espaciadores intersomáticos. Neurocirugia 2001; 12: 210.

14. Mobbs, R.J., Gollapudi, P.R., Chandran, N.K.: Outcome following anterior cervical discectomy in compensation patients. J Clin Neurosci 2001; 8: 124-125.

15. Odom, G.L., Finney, W., Woodhall, B.: Cervical disc lesions. JAMA 1958; 166: 23-28.

16. Porras-Estrada, L.F., Ugarriza Echeberrieta, L.F., Lorenzana-Honrado, L., et al.: Estudio comparativo de la artrodesis cervical con injerto óseo roscado e implante de titanio. Neurocirugia 2004; 15: 270-278.

17. R. Martín, Carda, J.R. Pinto, J.I. et al.: Discectomía cervical y artrodesis intersomática mediante técnica de Cloward: Análisis retrospectivo de las complicaciones y los resultados radiológicos en 167 casos. Neurocirugia 2002; 13 : 265-284.

18. Smith, G.W., Robinson, R.A.: The treatment of certain cervical-spine disorders by anterior removal of the intervertebral disc and interbody fusion. J Bone Joint Surg- A 1958; 40-A: 607-624.

19. Teo, E.C., Yang, K., Fuss, F.K., et al.: Effects of cervical cages on load distribution of cancellous core: a finite element analysis. J Spinal Disord Tech 2004; 17: 226-231.

20. Vavruch, L., Hedlund, R., Javid, D., Leszniewski, W., Shalabi, A.: A prospective randomized comparison between 
the cloward procedure and a carbon fiber cage in the cervical spine: a clinical and radiologic study. Spine 2002; 27: 16941701.

21. Wang, J.C., Yu, W.D., Sandhu, H.S., Tam, V., Delamarter, R.B.: A comparison of magnetic resonance and computed tomographic image quality after the implantation of tantalum and titanium spinal instrumentation. Spine 1998; 23: 16841688 .

22. Wigfield, C., Robertson, J., Gill, S., Nelson, R.: Clinical experience with porous tantalum cervical interbody implants in a prospective randomized controlled trial. $\mathrm{Br} \mathrm{J}$ Neurosurg 2003; 17: 418-425.

23. Zoega, B., Karrholm, J., Lind, B.: Outcome scores in degenerative cervical disc surgery. Eur Spine J 2000; 9: 137143.

24. Zung, W.W.K.: A self-rating depression scale. Arch Gen Psychiatry 1965; 12: 63-70.

Vicario, C.; Lopez-Oliva, F.; Sánchez Lorente, T.; Zimmermann, M.; Asenjo Siguero, J.J.; Ladero, F.; Ibarzábal, A.: Artrodesis cervical anterior mediante implante de tantalio. Resultados clínicos y radiológicos. Neurocirugía 2006; 17 : 132-139.

Correspondencia postal: Carlos Vicario Espinosa. C/ El Bosque 10. Cobisa, Toledo 45111.

\section{Comentario al artículo Artrodesis cervical anterior mediante implante de tantalio. Aspectos clínicos y radiológicos de $\mathbf{C}$. Vicario y cols.}

Este artículo proporciona resultados favorables con la introducción del tantalio poroso como prótesis cervical en las artrodesis cervicales, al menos similares a los registrados en la literatura con otros injertos. La serie de 24 pacientes analiza los parámetros clínicos y radiológicos descritos y muestra resultados positivos con cerca del $75 \%$ de resultados excelentes o buenos y un $85 \%$ de índice de fusión.

Los hallazgos iniciales son alentadores y deberían cumplimentarse con los mismos parámetros estudiados en seguimientos más largos. La posible limitación del estudio es el sesgo de selección, ya que más de la mitad de estos pacientes habían sufrido accidentes laborales, y las implicaciones clínicas que pueden derivarse de ello pueden hacer que los datos no sean extrapolables a la población general. Considerando los resultados obtenidos tanto clínicos como biomecánicos que resultan análogos a otras técnicas, y la ventaja que representan estos implantes respecto al titanio por la menor distorsión que ocasionan en la RM, la artrodesis cervical con tantalio se muestra como una técnica prometedora cuya utilidad deberá reevaluarse en estudios más extensos.

O. Mateo Madrid 